\title{
Preconditioned MLFMA Solution of Multiple Dielectric-Metallic Composite Objects with the Electric and Magnetic Current Combined-Field Integral Equation (JMCFIE)
}

\author{
Özgür Ergül ${ }^{1,2}$ and Levent Gürel ${ }^{1,2 *}$ \\ ${ }^{1}$ Department of Electrical and Electronics Engineering \\ ${ }^{2}$ Computational Electromagnetics Research Center (BiLCEM) \\ Bilkent University, TR-06800, Bilkent, Ankara, Turkey \\ \{ergul,lgurel\}@ee.bilkent.edu.tr
}

\section{Introduction}

We consider fast and accurate solutions of scattering problems involving multiple dielectric and composite dielectric-metallic structures with three-dimensional arbitrary shapes. Problems are formulated rigorously with the electric and magnetic current combined-field integral equation (JMCFIE) [1], which produces well-conditioned matrix equations. Equivalent electric and magnetic surface currents are discretized by using the Rao-Wilton-Glisson (RWG) functions defined on planar triangles. Matrix equations obtained with JMCFIE are solved iteratively by employing a Krylov subspace algorithm, where the required matrixvector multiplications are performed efficiently with the multilevel fast multipole algorithm (MLFMA) [2]. We also present a four-partition block-diagonal preconditioner (4PBDP), which provides efficient solutions of JMCFIE by reducing the number of iterations significantly. The resulting implementation based on JMCFIE, MLFMA, and 4PBDP is tested on large electromagnetics problems.

\section{JMCFIE Formulation and Its Discretization}

Consider the general case involving $U$ regions, namely, $D_{0}, D_{1}, \cdots, D_{U-1}$, and $D_{0}$ is a region extending to infinity. Each region $D_{u}$ for $u=0,1, \cdots,(U-1)$ is either metallic with perfect conductivity or lossless dielectric with constant electromagnetic parameters, i.e., permittivity $\epsilon_{u}$ and permeability $\mu_{u}$. Scattered electric and magnetic fields ( $\boldsymbol{E}^{s c a}$ and $\boldsymbol{H}^{s c a}$ ) in a nonmetallic medium $D_{u}$ can be obtained from the equivalent electric and magnetic currents $(\boldsymbol{J}$ and $\boldsymbol{M})$ defined on its surface $S_{u}$, i.e.,

$$
\begin{aligned}
\boldsymbol{E}^{s c a}(\boldsymbol{r}) & =\eta_{u} \mathcal{T}_{u}\{\boldsymbol{J}\}(\boldsymbol{r})-\mathcal{K}_{P V, u}\{\boldsymbol{M}\}(\boldsymbol{r})+\frac{\Omega_{i}}{4 \pi} \hat{\boldsymbol{n}} \times \boldsymbol{M}(\boldsymbol{r}) \\
\boldsymbol{H}^{s c a}(\boldsymbol{r}) & =\frac{1}{\eta_{u}} \mathcal{T}_{u}\{\boldsymbol{M}\}(\boldsymbol{r})+\mathcal{K}_{P V, u}\{\boldsymbol{J}\}(\boldsymbol{r})-\frac{\Omega_{i}}{4 \pi} \hat{\boldsymbol{n}} \times \boldsymbol{J}(\boldsymbol{r}) .
\end{aligned}
$$

where $0 \leq \Omega_{i} \leq 4 \pi$ is the internal solid angle, which is nonzero when the observation point $\boldsymbol{r}$ is on the surface, $\eta_{u}=\sqrt{\mu_{u} / \epsilon_{u}}$ and $\hat{\boldsymbol{n}}$ is the outward normal unit vector.

For numerical solutions, JMCFIE is discretized with the RWG functions using a Galerkin scheme. Basis functions are indexed by firstly considering the nonmetallic surfaces, which involve $N_{D} \leq N$ basis functions. The remaining $\left(N-N_{D}\right)$ basis functions, if any, are defined on metallic surfaces to expand the electric current. Discretization of JMCFIE leads

\footnotetext{
${ }^{\dagger}$ This work was supported by the Scientific and Technical Research Council of Turkey (TUBITAK) under Research Grants 105E172 and 107E136, by the Turkish Academy of Sciences in the framework of the Young Scientist Award Program (LG/TUBA-GEBIP/2002-1-12), and by contracts from ASELSAN and SSM.
} 
to $\left(N+N_{D}\right) \times\left(N+N_{D}\right)$ dense matrix equations in the form of

$$
\left[\begin{array}{ll}
{\left[\overline{\boldsymbol{Z}}_{11}\right]_{N \times N}} & {\left[\overline{\boldsymbol{Z}}_{12}\right]_{N \times N_{D}}} \\
{\left[\overline{\boldsymbol{Z}}_{21}\right]_{N_{D} \times N}} & {\left[\overline{\boldsymbol{Z}}_{22}\right]_{N_{D} \times N_{D}}}
\end{array}\right] \cdot\left[\begin{array}{l}
\boldsymbol{a}^{(1)} \\
\boldsymbol{a}^{(2)}
\end{array}\right]=\left[\begin{array}{l}
\boldsymbol{v}^{(1)} \\
\boldsymbol{v}^{(2)}
\end{array}\right],
$$

where $\boldsymbol{a}^{(1)}$ and $\boldsymbol{a}^{(2)}$ are vectors of $N$ and $N_{D}$ elements involving the coefficients of the electric and magnetic currents, respectively. In (3), matrix elements are calculated by considering the interactions of the basis and testing functions through nonmetallic regions. As an example, consider the interaction between a basis function $\boldsymbol{b}_{n}$ and a testing function $\boldsymbol{t}_{m}$ for $n \leq N$ and $m \leq N$, and let a dielectric region $D_{u}$ be common for the two functions. A contribution to the matrix element in the diagonal partition $\bar{Z}_{11}$ can be written as

$$
\begin{aligned}
\overline{\boldsymbol{Z}}_{11}[m, n] \stackrel{+}{\longleftarrow} & -\frac{\xi_{n} \xi_{m}}{2} \int_{S_{m}} d \boldsymbol{r} \boldsymbol{t}_{m}(\boldsymbol{r}) \cdot \boldsymbol{b}_{n}(\boldsymbol{r})+\xi_{n} \int_{S_{m}} d \boldsymbol{r} \boldsymbol{t}_{m}(\boldsymbol{r}) \cdot \hat{\boldsymbol{n}} \times \mathcal{K}_{P V, u}\left\{\boldsymbol{b}_{n}\right\}(\boldsymbol{r}) \\
& +\xi_{n} \xi_{m} \int_{S_{m}} d \boldsymbol{r} \boldsymbol{t}_{m}(\boldsymbol{r}) \cdot \mathcal{T}_{u}\left\{\boldsymbol{b}_{n}\right\}(\boldsymbol{r})
\end{aligned}
$$

where the signs $\xi_{m}= \pm 1$ and $\xi_{n}= \pm 1$ are determined by the orientation of the basis and testing functions. When the basis function $\boldsymbol{b}_{n}$ is not on a metallic surface, i.e., $n \leq N_{D}$,

$$
\begin{aligned}
\overline{\boldsymbol{Z}}_{12}[m, n] \stackrel{+}{+} & -\frac{\xi_{n}}{2 \eta_{u}} \int_{S_{m}} d \boldsymbol{r} \boldsymbol{t}_{m}(\boldsymbol{r}) \cdot \hat{\boldsymbol{n}} \times \boldsymbol{b}_{n}(\boldsymbol{r})-\frac{\xi_{n} \xi_{m}}{\eta_{u}} \int_{S_{m}} d \boldsymbol{r} \boldsymbol{t}_{m}(\boldsymbol{r}) \cdot \mathcal{K}_{P V, u}\left\{\boldsymbol{b}_{n}\right\}(\boldsymbol{r}) \\
& +\frac{\xi_{n}}{\eta_{u}} \int_{S_{m}} d \boldsymbol{r} \boldsymbol{t}_{m}(\boldsymbol{r}) \cdot \hat{\boldsymbol{n}} \times \mathcal{T}_{u}\left\{\boldsymbol{b}_{n}\right\}(\boldsymbol{r})
\end{aligned}
$$

Similarly, when the testing function $\boldsymbol{t}_{m}$ is not on a metallic surface, i.e., $m \leq N_{D}$,

$$
\begin{aligned}
\overline{\boldsymbol{Z}}_{21}[m, n] \stackrel{+}{\longleftarrow} & \frac{\xi_{n}}{2} \eta_{u} \int_{S_{m}} d \boldsymbol{r} \boldsymbol{t}_{m}(\boldsymbol{r}) \cdot \hat{\boldsymbol{n}} \times \boldsymbol{b}_{n}(\boldsymbol{r})-\xi_{n} \eta_{u} \int_{S_{m}} d \boldsymbol{r} \boldsymbol{t}_{m}(\boldsymbol{r}) \cdot \hat{\boldsymbol{n}} \times \mathcal{T}_{u}\left\{\boldsymbol{b}_{n}\right\}(\boldsymbol{r}) \\
& +\xi_{n} \xi_{m} \eta_{u} \int_{S_{m}} d \boldsymbol{r} \boldsymbol{t}_{m}(\boldsymbol{r}) \cdot \mathcal{K}_{P V, u}\left\{\boldsymbol{b}_{n}\right\}(\boldsymbol{r}) .
\end{aligned}
$$

Finally, when both basis and testing functions are not on metallic surfaces, there exists a matrix element $\overline{\boldsymbol{Z}}_{22}[m, n]=\overline{\boldsymbol{Z}}_{11}[m, n]$. Elements of the the excitation vectors $\boldsymbol{v}^{(1)}$ and $\boldsymbol{v}^{(2)}$ in (3) are calculated by testing the incident electric and magnetic fields due to external sources.

\section{Multilevel Fast Multipole Algorithm}

In iterative solutions of JMCFIE, we employ MLFMA to perform matrix-vector multiplications in $\mathcal{O}(N \log N)$ time using $\mathcal{O}(N \log N)$ memory. Each nonmetallic region is included in a cubic box and recursively divided into clusters. Then, MLFMA calculates the distant interactions between the basis and testing functions in a group-by-group manner consisting of three stages, called aggregation, translation, and disaggregation, which are performed on each tree structure in a multilevel scheme. In an aggregation stage for a nonmetallic region, radiated fields of the clusters are calculated from the bottom of the tree structure to the highest level. In the lowest level, radiation patterns of the RWG functions, which are located on the surface of the region, are multiplied with the coefficients provided by the iterative algorithm, and they are combined to obtain the radiated fields of the lowest-level clusters. Radiated field of a cluster in a higher level is obtained by combining the radiated fields of 
its subclusters. In the translation stage, radiated fields are translated into incoming fields. Then, in the disaggregation stage, total incoming fields at cluster centers are calculated from the top of the tree structure to the lowest level. In the lowest level, incoming fields are received by the testing functions. Similar to the aggregation stage, the disaggregation stage for a region involves only the RWG functions located on the surface of the region.

\section{Four-Partition Block-Diagonal Preconditioner}

MLFMA is required but not sufficient for efficient solutions of JMCFIE. The number of iterations should also be small, in addition to fast matrix-vector multiplications. Although JMCFIE is a second-kind integral equation and it provides well-conditioned matrix equations, iterative solutions of JMCFIE can be difficult, especially when the problem size is large. To improve the iterative solutions of JMCFIE, we propose 4PBDP, which is based on using self interactions of the lowest-level clusters, i.e.,

$$
\overline{\boldsymbol{P}}_{4 P}=\left[\begin{array}{cc}
\overline{\boldsymbol{P}}_{N \times N}^{(11)} & \overline{\boldsymbol{P}}_{N \times N_{D}}^{(12)} \\
\overline{\boldsymbol{P}}_{N_{D} \times N}^{(21)} & \overline{\boldsymbol{P}}_{N_{D} \times N_{D}}^{(22)}
\end{array}\right] \approx\left[\begin{array}{cc}
\overline{\boldsymbol{Z}}_{N \times N}^{(11)} & \overline{\boldsymbol{Z}}_{N \times N_{D}}^{(12)} \\
\overline{\boldsymbol{Z}}_{N_{D} \times N}^{(21)} & \overline{\boldsymbol{Z}}_{N_{D} \times N_{D}}^{(22)}
\end{array}\right]
$$

Processing time required for extracting, inverting, and applying $\overline{\boldsymbol{P}}_{4 P}$ are $\mathcal{O}(N)$.

\section{Results}

Efficiency and accuracy of the resulting JMCFIE-MLFMA-4PBDP implementation are demonstrated on various electromagnetics problems involving relatively large objects. As an example, Fig. 1(a) presents iteration counts for the solution of scattering problems involving a composite spherical object. A dielectric core with a radius of $a$ is coated with a shell of radius $2 a$, and $a$ changes from $0.5 \lambda_{0}$ to $2.5 \lambda_{0}$, where $\lambda_{0}$ is the wavelength outside the object (free space). Relative permittivities of the core and the shell are 2.0 and 4.0, respectively. The object is illuminated by a plane wave propagating in the $-x$ direction with the electric field polarized in the $y$ direction. Surfaces are discretized with about $\lambda_{0} / 10$ mesh size leading to matrix equations with 13,176 to 316,032 unknowns. Fig. 1(a) depicts the number of biconjugate-gradient-stabilized (BiCGStab) iterations to reach $10^{-3}$ residual error as a function of the number of unknowns. We observe that 4PBDP accelerates the iterative solutions significantly compared to the no-preconditioner (NP) case. When $a=1.67 \lambda_{0}$, convergence cannot be achieved in 1000 iterations without preconditioning. Using 4PBDP, the number of iterations is less than 100 for all solutions in Fig. 1(a).

Fig. 1(b) presents the solution of a larger problem involving a coated metallic sphere. A metallic core with a radius of $5 \lambda_{0}$ is coated with a dielectric shell of radius $10 \lambda_{0}$, where $\lambda_{0}$ is the wavelength outside the object (free space). Relative permittivity of the shell is 4.0 . The object is again illuminated by a plane wave propagating in the $-x$ direction with the electric field polarized in the $y$ direction. Discretization of the surfaces with about $\lambda_{0} / 10$ mesh size leads to a matrix equation with $1,264,128$ unknowns. The problem is solved by employing a 6-level MLFMA with two digits of accuracy. With the acceleration provided by 4PBDP, the number of BiCGStab iterations to reach $10^{-3}$ residual error is 187. Fig. 1(b) depicts normalized bistatic radar cross section $\left(\mathrm{RCS} / \lambda_{0}^{2}\right)$ in decibels $(\mathrm{dB})$ as a function of the observation angle on the $\phi=0^{\circ}$ plane, where $0^{\circ}$ corresponds to the forward-scattering direction. We observe that the computational results obtained with JMCFIE-MLFMA-4PBDP are in agreement with the analytical results obtained by a Mie-series solution. 


\section{References}

[1] P. Ylä-Oijala and M. Taskinen, "Application of combined field integral equation for electromagnetic scattering by dielectric and composite objects," IEEE Trans. Antennas Propagat., vol. 53, no. 3, pp. 1168-1173, Mar. 2005.

[2] J. Song, C.-C. Lu, and W. C. Chew, "Multilevel fast multipole algorithm for electromagnetic scattering by large complex objects," IEEE Trans. Antennas Propagat., vol. 45, no. 10, pp. 1488-1493, Oct. 1997.

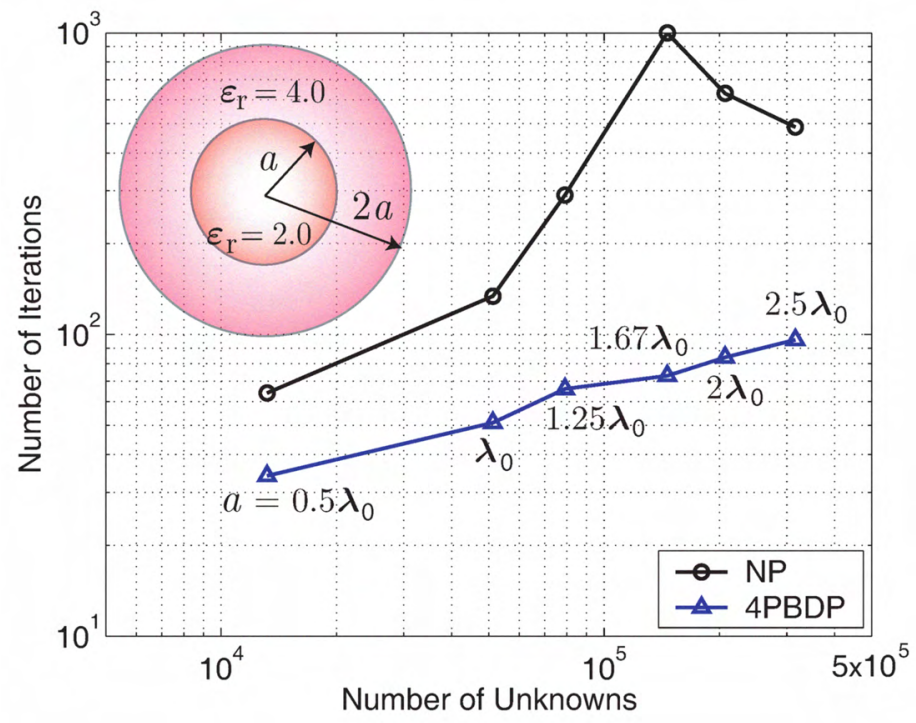

(a)

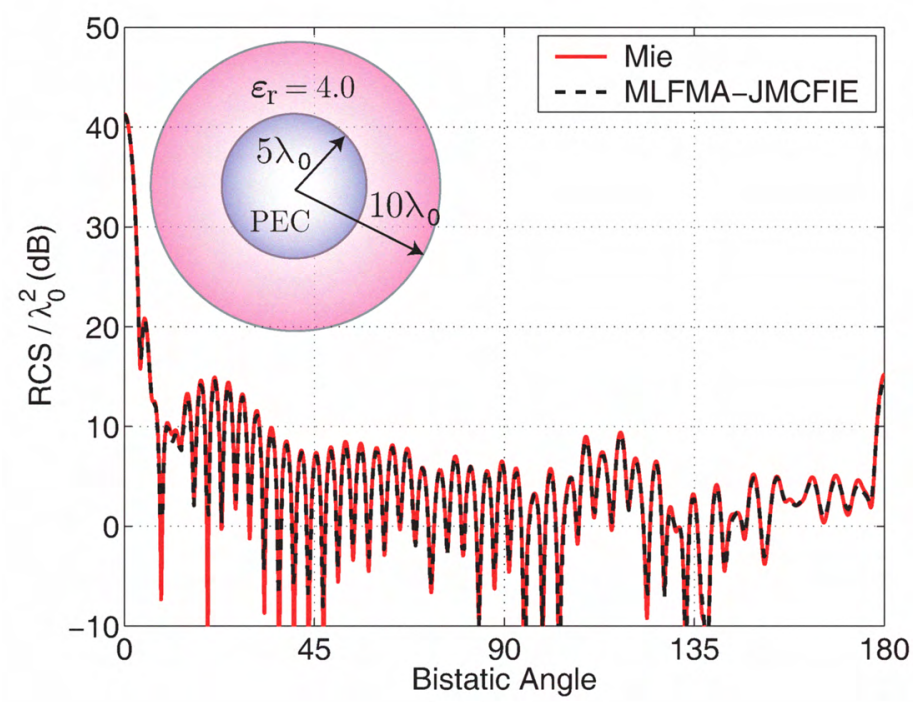

(b)

Fig. 1. (a) Iteration counts for the solution of scattering problems involving a dielectric sphere of radius $a$ coated with a dielectric shell of radius $2 a$, where $a$ changes from $0.5 \lambda_{0}$ to $2.5 \lambda_{0}$. (b) Normalized bistatic RCS $\left(\operatorname{RCS} / \lambda_{0}^{2}\right)$ of a metallic sphere of radius $5 \lambda_{0}$ coated with a dielectric shell of radius $10 \lambda_{0}$. 